\title{
STATIC AND DYNAMIC EVALUATION OF THE DRIVER SPEED PERCEPTION AND SELECTION PROCESS
}

\author{
David S. Hurwitz, Michael A. Knodler, Jr. \\ University of Massachusetts Amherst \\ Department of Civil \& Environmental Engineering \\ Amherst, Massachusetts, United States \\ E-mail: dhurwitz@student.umass.edu \\ E-mail: mknodler@ecs.umass.edu
}

\begin{abstract}
Summary: Speed impacts the extent to which mobility and safety are experienced across the surface transportation network. By expanding current understanding of speed perception and selection processes our ability to understand and comprehensively address speed-related issues will improve. Driving simulator technology has advanced the field of transportation research. However, it has been limited in its application to speed-related issues. Furthermore, static computer-based evaluations have been used as a means of establishing a preliminary understanding for driver interpretation of stimuli encountered in the roadway, but have been limited in their application to speed. These technologies allow for large sample populations to be evaluated quickly and safely. Phase I of this initiative examined driver ability to perceive travel speeds in a similar real world, simulated world, and static environment. The experimental course traversed roadway where land-use and posted speed limits varied. Drivers' actual and perceived speeds were recorded at 20 identical "checkpoint" locations in each environment, and the results were analyzed across drivers and environments. Phase II examined three roadway attributes that impact the speed-selection process. A focus group was employed to build improved scenarios of interest for a full-scale static evaluation. In the static environment, 75 drivers were asked how fast they would travel while individual characteristics of the scenario displayed were modified. This multifaceted research initiative expands the potential application of advanced technology in speed-related research, and improves the understanding of factors that influence speed perception and selection processes.
\end{abstract}

\section{INTRODUCTION}

Roadway speed is consistently a critical element in traffic operations and safety, and as a result has become the focus of numerous research efforts. From a safety perspective, speed is regularly attributed to be one of the greatest detriments to roadway safety. In 2005, the National Highway Traffic Safety Administration (NHTSA) estimated that speed was a contributing factor in approximately 30 percent of all fatal crashes, accounting for 13,113 fatalities at an estimated cost of $\$ 40.4$ billion dollars (National Center for Statistics and Analysis, 2005). As a result, all facets of speed, including the processes by which drivers select and are able to accurately perceive a travel speed, are of concern to transportation professionals. 
Questions remain as to the manner in which drivers select a given travel speed. A number of variables contribute to drivers' speed selection, articulating each is a monumental task; however, using a number of "grouped" real-world scenarios to determine what environmental factors impact the speed-selection process would benefit researchers, transportation professionals and law enforcement officials.

A need exists to foster a greater understanding of the driver speed perception and selection processes. This high level of understanding would be invaluable to those responsible for all facets of roadway safety, including but not limited to, roadway designers, speed enforcement personnel, and safety stakeholders. Furthermore, this understanding would also be critical in the development of optimal solutions for problems pertaining to speed management that may not be achieved until these processes are understood to a greater extent. This research initiative addressed the potential use of experimental environments generated with advanced technology (i.e., driving simulator and laptop-based static) to facilitate speed-related research. If these technologies can be validated, transportation researchers will have the ability to evaluate various scenarios with larger sample sizes in a cost-effective, efficient, and safe environment.

\section{EXPERIMENT}

\section{Phase I}

Field. In 2005, Knodler and Dulaski completed a field-based study that considered the driver speed-selection process as a function of a roadway's functional classification (Knodler \& Dulaski, 2005). Although the study was primarily centered upon the speeds along roadways of varying functional classifications and subsequently, speed limits, additional roadway and demographic elements were also considered. The raw data from this earlier experiment was directly incorporated into this experiment; however, all of the analyses are unique to this effort.

Driving simulator. The driving simulator evaluation was conducted with the use of a fixed-base, fully-interactive, dynamic driving simulator, housed in the Human Performance Laboratory (HPL) at the University of Massachusetts. The simulator includes a fully instrumented 1995 Saturn Sedan, three mounted overhead projectors, three projection screens, and a Bose surround sound audio system. The projected images that make up the virtual roadway adjust according to the drivers' actions. The visual road is composed of 3 separate images projected onto the screens in front of the vehicle, producing a 150-degree semicircular field-of-view. The images produced are refreshed at a rate of $60 \mathrm{~Hz}$ and have a resolution of 1024 x $768 \mathrm{dpi}$. In the simulator, drivers control steering, braking, and accelerating in a fashion similar to what drivers would expect in the field.

The simulated environment experienced by drivers was an exact virtual replica of the three-mile course used in the field experiment. Furthermore, the same drivers who were evaluated in the field experiment participated in the simulator experiment. The level of replication was intended to promote realism and similarity to the original field experiment. Functional classifications were addressed by identifying pavement widths that appear to be the same as those in the real environment, as well as the correct pavement markings. Adjacent land use was replicated by 
reproducing the magnitude and location of houses, as well as significant landmarks (i.e., large trees, mailboxes, etc.) that appeared in close proximity to the roadway.

The same 20 "checkpoint" locations were established in the simulated environment consistent with Figure 1. The instrument panel was occluded from the drivers' view, and drivers were asked to state their perceived speed travel at each checkpoint. This information was recorded by research personnel and verified with simulator output data.

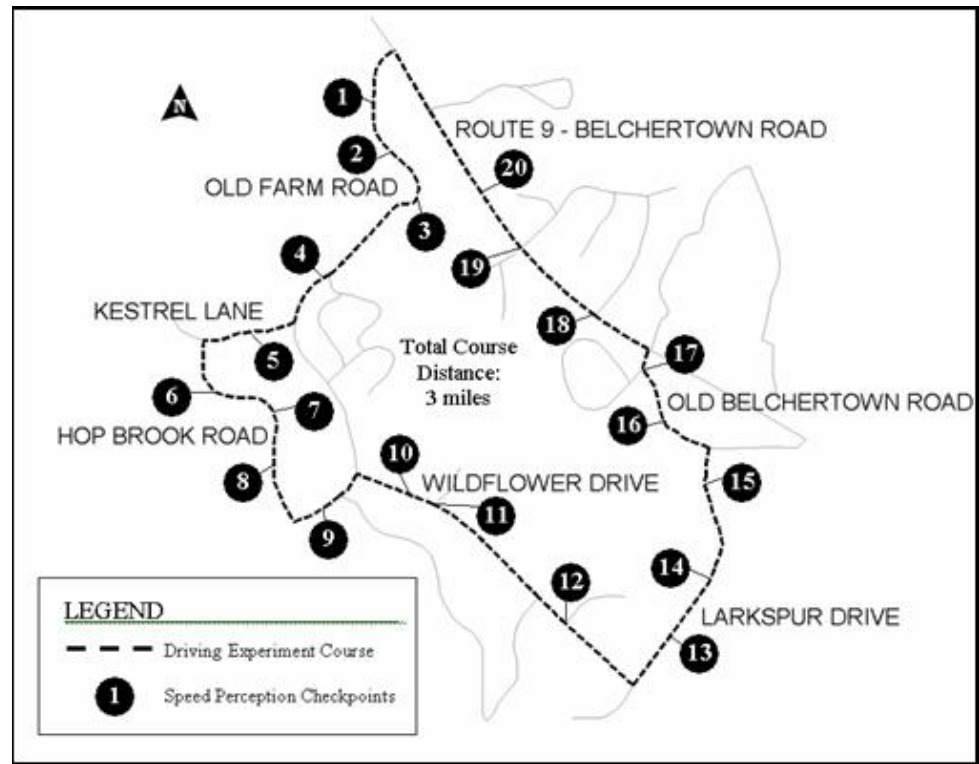

Figure 1. Checkpoint Locations of Field, Simulator, and Computer-Based Experiments

To promote the capture of free-flow speeds, the checkpoints were established at locations that would not be unduly influenced by the proximity of intersections or other vehicles. Lead vehicles, which are often used in the simulation to guide drivers, would influence the free-flow speed of the drivers. Therefore, researchers directed drivers in the vehicle consistent with the onroad experiment (Hurwitz, Knodler, \& Dulaski, 2005)

Follow-up Static. The follow-up static evaluation was consistent with the scenarios presented in both the field and driving simulator. Specifically, the evaluation was developed and administered on a laptop computer and was composed of scenarios incorporating real-world images captured from each of 20 "checkpoint" locations on the roadway consistent with both the field and simulator portions of the research. Driver responses were recorded by the driver on separate response sheets and reduced for analysis purposes. Two questions were presented in conjunction with each image: "What speed would you travel on the roadway shown?"

\section{Phase II}

Focus Group. The focus group experiment was included to improve the structure and content of the scenarios presented in the expanded computer-based static evaluation, as well as to provide further insight on the research questions. A single focus group of 8 participants was assembled 
with the primary concern being an examination of driver perspectives on speed selection as influenced by pavement markings.

The focus group was composed of younger male and female drivers and was designed to present ,19 target questions in a one-hour period. The structure of the focus was such that drivers were first introduced to the topic (10 minutes), asked general questions about factors that influence speeding behavior (20 minutes), and asked about the impact of pavement markings on speed selection (30 minutes).

Full-Scale Static Evaluation. A full scale static evaluation examined the influence of assorted pavement marking configurations on the driver speed-selection process. The configurations were established by manipulating four variables: functional classification, center line pattern, edge line, and adjacent barriers. These variables were identified by the focus group as having a significant influence on the speed selection process. An attempt was made to randomize the presentation of scenarios by administering five distinct iterations of the static evaluation, with each iteration presenting 21 developed scenarios. Table 1 identifies all of the scenarios included based on the number of degrees with which each variable was examined.

\section{Table 1. Scenarios Presented in the Full-Scale Static Evaluation}

\section{RESULTS}

\begin{tabular}{|c|c|c|c|c|}
\hline $\begin{array}{c}\text { Functional } \\
\text { Classification }\end{array}$ & $\begin{array}{c}\text { Center Line } \\
\text { (CL) }\end{array}$ & $\begin{array}{l}\text { Edge Line } \\
\text { (EL) }\end{array}$ & Barrier & $\begin{array}{c}\text { Scenario } \\
\text { Identification }\end{array}$ \\
\hline \multirow{6}{*}{ Interstate } & \multirow{3}{*}{$S_{Y L}{ }^{a}$} & \multirow{3}{*}{$2 \mathrm{ft}$} & Jersy Barrier & $11^{e}$ \\
\hline & & & Guard Rail & $12^{e}$ \\
\hline & & & Not Present & $13^{e}$ \\
\hline & \multirow{3}{*}{$\mathrm{BWLL}^{b}$} & \multirow{3}{*}{$6 \mathrm{ft}$} & Jersy Barrier & $14^{f}$ \\
\hline & & & Guard Rail & $15^{f}$ \\
\hline & & & Not Present & $16^{f}$ \\
\hline \multirow{6}{*}{ Arterial } & \multirow{6}{*}{$\mathrm{DYL}^{c}$} & \multirow{3}{*}{$2 \mathrm{ft}$} & Jersy Barrier & A1 \\
\hline & & & Guard Rail & A2 \\
\hline & & & Not Present & A3 \\
\hline & & \multirow{3}{*}{$6 \mathrm{ft}$} & Jersy Barrier & A4 \\
\hline & & & Guard Rail & A5 \\
\hline & & & Not Present & A6 \\
\hline \multirow{6}{*}{ Collector } & $\mathrm{DYL}^{c}$ & \multirow{2}{*}{$6 \mathrm{ft}$} & \multirow{2}{*}{ Not Present } & C1 \\
\hline & $\mathrm{BYCL}^{d}$ & & & $\mathrm{C} 2$ \\
\hline & $\mathrm{DYL}^{c}$ & \multirow{2}{*}{$2 \mathrm{ft}$} & \multirow{2}{*}{ Not Present } & $\mathrm{C} 3$ \\
\hline & $\mathrm{BYCL}^{d}$ & & & $\mathrm{C} 4$ \\
\hline & $\mathrm{DYL}^{c}$ & Not Present & Not Present & C5 \\
\hline & $\mathrm{BYCL}^{d}$ & IVot riesem & Notriesemi & C6 \\
\hline \multirow{3}{*}{ Local } & $\mathrm{DYL}^{c}$ & \multirow{3}{*}{ Not Present } & \multirow{3}{*}{ Not Present } & L1 \\
\hline & $S Y L^{a}$ & & & $\mathrm{~L} 2$ \\
\hline & Not Present & & & L3 \\
\hline \multicolumn{5}{|l|}{${ }^{a}$ Solid Yellow Line } \\
\hline \multicolumn{5}{|c|}{${ }^{b}$ Broken White Lane Line } \\
\hline \multicolumn{5}{|c|}{${ }^{c}$ Double Yellow Line } \\
\hline \multicolumn{5}{|c|}{${ }^{d}$ Broken Yellow Center Line } \\
\hline \multicolumn{5}{|c|}{${ }^{e}|1|$,2 , and $I 3$ take place in the left lane of an Interstate } \\
\hline
\end{tabular}

\section{Phase I}

The comparison of the mean actual speeds recorded in the simulator and field are displayed in Figure 2. Similar general trends in actual speed were observed across all 20 checkpoints. 
Generally, the mean speed in the simulator was greater than that in the field. The mean of the predicted speeds from the static was less than the mean of the actual speed from the field at checkpoints 2, 4, 8, and 19. Checkpoints 2, 4, and 8 occur on local roads. In instances where a local maximum appears to occur, the mean speed of travel in the simulator is significantly higher than that of the field.

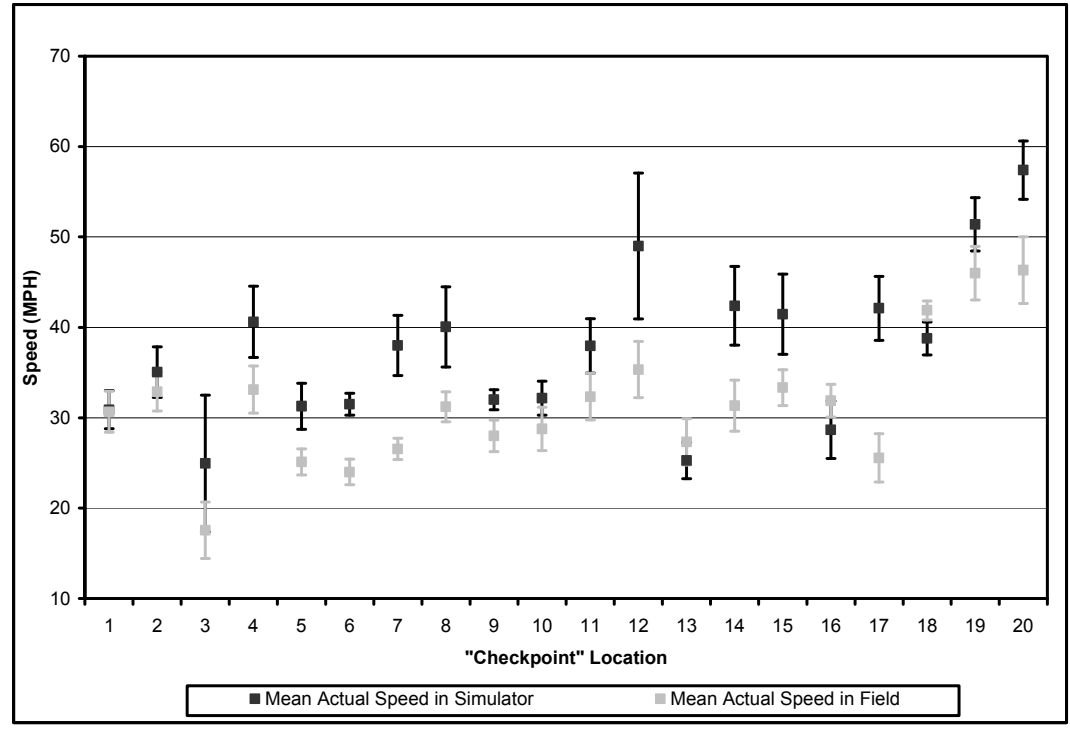

Figure 2. Mean Actual Speed in Field and Simulator with 95\% C.I.

\section{Phase II}

Focus Group. Eight drivers (three males and five females) participated in the focus group experiment. The mean age for the group was 26.5, and the mean years of driving experience was 10.2. Consistent with Phase I, the recorded demographics again include age, sex, driving experience, and miles driven in the previous year.

It was determined that drivers consciously perceive pavement markings on the roadway, and that the markings influenced the speeds at which they drove. However they did not think that these markings were as influential as other controlling factors, such as the environment or the amount of experience that they had driving in a particular area.

The data presented here represent a sample of responses generated from the focus group, which were used to provide a further understanding of the relative importance that drivers place on the engineered attributes of the roadway in the speed-selection process. The data also helped to refine several series of testable scenarios in the full-scale static evaluation.

- In response to the question, "Do you consciously notice pavement markings" the unanimous consensus was that pavement markings are consciously noticed in a variety of situations.

- In response to the question, "Do you think that pavement markings influence the speed at which you drive?" it was the consensus of the respondents that good lane markings make drivers more comfortable, and probably have more of an impact on narrow roads and at night on highways. 
- In response to the question, "Do you think that markings at the shoulder of the road affect your speed?" several respondents felt that they would impact the speed of travel and that they probably cause them to slow down.

In summary, three of the variables included in the full-scale static evaluation are lane width, shoulder markings, and jersey barriers / guard rails. Based upon the focus group data, an expectation for the static evaluation would be reduced speeds when drivers are presented with narrow lanes, marked shoulders, or adjacent jersey barriers, and that wide lanes have a negligible impact on travel speeds.

Full-Scale Static Evaluation. Seventy-five drivers (26 males and 49 females) participated in the static experiment. The average predicted speed of travel with $95 \%$ confidence intervals was been calculated for each of the 21 scenarios. This data was then aggregated by functional classification into bar graphs that display predicted speed on the y-axis and describe the variables degree on the $x$-axis. An examination of the results in this manner provided insight into the influence of particular pavement marking configurations on the speed-selection process with a functional class of roadway. It also provided a means of comparing different functional class roadways. Results from this experiment appear in Figure 3.

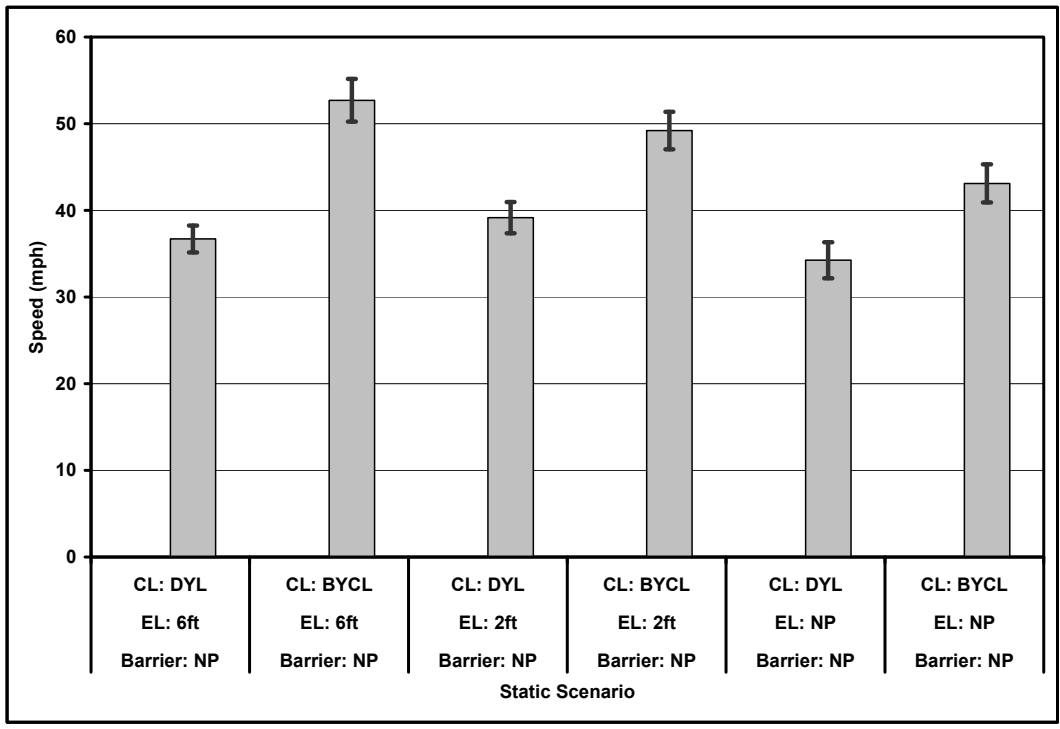

Figure 3. Drivers' Average Predicted Speed for the Collector Scenarios

Preliminary evidence suggests with $95 \%$ confidence that drivers predicted travel speed on a collector type roadway with a 6-ft shoulder, a 2-ft shoulder, or no shoulder present is faster with a broken yellow center line than with a double yellow line. There is also evidence to suggest that the average predicted speed of travel with a broken yellow centerline is faster on a 6-ft shoulder than when a shoulder is not present.

\section{CONCLUSIONS}

The study allowed for a preliminary comparison of driver speed perception in a field and driving simulator environment. The purpose was to expand upon the existing understanding of the driver 
speed-perception process, and to evaluate the potential application of driving simulator technology in speed-related research. Based on the findings, there is initial evidence to suggest that drivers tend to underestimate their travel speeds in both environments. Also, there appears to be a consistency in the trends associated with both the speeds selected and perceived. For example, in both environments drivers were operating and perceiving higher speeds on roadways with higher speed limits. Additional conclusions include the following:

- Based on initial findings it appears that drivers are more accurate perceiving their travel speed in the field. On average, the order of magnitude is approximately $5 \mathrm{mph}$ difference in the simulator versus the field.

- It is also apparent that driver performance was affected at certain "checkpoint" locations. An initial inspection draws a strong correlation between horizontal curvature and speed perception. Although drivers tended to have greater difficulty estimating speed on horizontal curves, this difference appears to be more pronounced in the driving simulator.

- It is apparent that speed perception is an attribute that varies between drivers, (i.e., some drivers are more accurate or precise in their perception of speed) regardless of the experimental medium employed.

- There is preliminary evidence suggesting that differences may exist in drivers' perceptions of speed along downgrades in the simulator environment.

The full-scale static evaluation was completed to address the following research objective, "Determine the magnitude with which assorted roadway characteristics impact speed selection." Several scenarios were identified to have statistically significant differences in average predicted speed from the other scenarios within the same functional classification of roadway. The following configurations were found to affect drivers' predicted speed of travel:

- With 95\% confidence the experimental variables did not impact the drivers' predicted speed of travel on the Interstate scenarios.

- On an arterial roadway with a DYL and a 2-ft shoulder, drivers predict a slower speed of travel with an adjacent guardrail, than in the presence of a jersey barrier or no barrier.

- On a collector roadway, drivers predict a higher speed of travel with a BYCL than with a DYL with a 2-ft, 6-ft, or no marked shoulder.

\section{REFERENCES}

Hurwitz, D., Knodler, M., \& Dulaski, D. (2005). Speed perception fidelity in a driving simulator environment. Proceedings of the Driving Simulator Conference - North America, Orlando, Florida, 343-352.

Knodler, Jr., M.A., \& Dulaski, D.M. (2005). An initial evaluation of the driver speed selection process on posted and unposted roadways varying in functional classification. Proceedings of the ITE Technical Conference and Exhibit, Las Vegas, Nevada.

National Center for Statistics and Analysis. (2005). Traffic safety facts 2005: Speeding. (DOT HS 810 629). Washington, DC: Department of Transportation. 